OPEN ACCESS

Edited by:

Sandip D. Kamath,

James Cook University, Australia

Reviewed by:

Ciaran Skerry,

University of Maryland, Baltimore,

United States

Pietro Speziale,

University of Pavia, Italy

${ }^{*}$ Correspondence:

Ali Azizi

ali.azizi@sanofi.com

Specialty section:

This article was submitted to

Vaccines and Molecular Therapeutics,

a section of the journal

Frontiers in Immunology

Received: 11 September 2020

Accepted: 11 November 2020

Published: 15 December 2020

Citation:

Yougbare I, McTague A, He L,

Choy $\mathrm{CH}$, Su J, Gajewska B and Azizi A (2020) Anti-FIM and Anti-FHA

Antibodies Inhibit Bordetella

pertussis Growth and Reduce

Epithelial Cell Inflammation

Through Bacterial Aggregation.

Front. Immunol. 11:605273.

doi: 10.3389/fimmu.2020.605273

\section{Anti-FIM and Anti-FHA Antibodies Inhibit Bordetella pertussis Growth and Reduce Epithelial Cell Inflammation Through Bacterial Aggregation}

\author{
Issaka Yougbare, Adam McTague, Liwei He, Christopher H. Choy, Jin Su,
} Beata Gajewska and Ali Azizi *

Immunology platform, Analytical Sciences North America, Sanofi Pasteur, Toronto, ON, Canada

The pertussis vaccination is highly recommended for infants, children, and pregnant women. Despite a high coverage of vaccination, pertussis continues to be of public health concern as a re-emerging infectious disease. The mechanism by which vaccine-elicited anti-pertussis antibodies mediate direct bactericidal effects is poorly understood. In this study, we showed that the interaction of B. pertussis with A549 epithelial cells induce release of biological factors which enhance bacteria growth. Complement-depleted antisera from vaccine-immunized guinea pigs or monoclonal antibodies targeting FHA and FIM mediate bacteria aggregation and elicit bactericidal effects. Our in vitro results indicated that aggregation of bacteria through anti-FIM and anti-FHA specific antibodies is one of the major biological mechanisms to clear bacterial infections and restore epithelial cell survival in vitro. Our data also indicates that the anti-pertussis antibodies reduce secretion of proinflammatory chemokines and cytokines by preventing interaction of $B$. pertussis with host cells. The results of this study not only demonstrate mechanism of action of anti-FIM and anti-FHA antibodies, but also opens translational applications for potential therapeutic approaches or development of analytical assays such as in vitro potency assays.

Keywords: anti-pertussis antibodies, aggregation, bacterial growth inhibition, epithelial inflammation, cytokines

\section{INTRODUCTION}

Pertussis, also known as whooping cough, is a re-emerging infectious disease in many countries despite high coverage of vaccines $(1,2)$. This life-threatening contagious disease is caused by the Gram-negative bacterium, Bordetella pertussis. Re-emergence of pertussis despite widespread vaccination is due to various parameters including the selection or evolution of mutant $B$. pertussis strains or waning protection of acellular vaccines (1,3-5). For an effective control of pertussis, it is recommended to strengthen vaccination coverage among the whole population by providing primary vaccination to newborns, and boosting infants and adults every 10 years (6). The recent re-emergence of the disease in North America showed that pertussis is particularly 
dangerous for infants under 4 months of age, accounting for $86 \%$ of all pertussis associated fatalities (7). Vaccination of women at the third trimester of pregnancy has been shown to be the most effective intervention to protect the newborn from pertussis (8). The passively transferred maternal antibodies to the neonates play a protective role before pertussis vaccination can be implemented $(9,10)$. Studies have shown strong humoral protection in infants; however, the mechanism by which antibodies control $B$. pertussis infection has not been well understood $(9,11,12)$.

Adverse reactions after immunization with the whole-cell pertussis ( $\mathrm{wP}$ ) vaccine led to the development of acellular pertussis (aP) vaccines with less reactogenicity (13). The aPbased vaccines contain up to five pertussis antigens including pertactin (PRN), filamentous hemagglutinin adhesin (FHA), fimbriae 2 and 3 (FIM), and pertussis toxoid (PT). Among several adhesion proteins produced by $B$. pertussis, FIM, and FHA have been shown to allow the bacterium to attach to host epithelial and immune cells. Agglutinogens FIM2 and FIM3 are key adhesion proteins with the molecular weight $22 \mathrm{kDa}$ and 22.5 $\mathrm{kDa}$ respectively. While FIM2 and FIM 3 are structurally related, they are serologically distinct (14). It has been suggested that FIMmediated adherence of $B$. pertussis to airway epithelium is a first step in infection, allowing adherence, suppressing inflammation, and enhancing resistance to inflammatory cell-mediated clearance (15). FHA is a $220 \mathrm{kDa}$ surface-associated protein that predominantly serves as an adhesion molecule to host ciliated epithelial cells, therefore FHA is associated with biofilm formation, and persistence of the infection (16-18). FHA possesses four binding domains which can bind to different cell receptors on the epithelial cell surface; its immune modulatory effects have also been reported on immune cells (19). PT and PRN have been also reported as adhesins, however, their roles in $B$. pertussis adhesion to host cells remain controversial $(20,21)$.

The mechanism underlining antibody mediated inhibition of bacterial adhesion to the epithelium and any potential bactericidal effect is poorly understood. The aim of this study was to explore the function of pertussis adhesion antigens (FHA and FIM) by inhibiting their interactions with host epithelial cells (A549) using antigen-specific antibodies. To achieve this, we have established a co-culture system with B. pertussis and A549 cells and used guinea pig polyclonal sera or mouse monoclonal antibodies (mAbs) against either FHA or FIM to study the blocking effect on interactions of $B$. pertussis with host cells. We have shown that anti-FHA and anti-FIM antibodies can inhibit B. pertussis adhesion to host cells and reduce the bacterial growth. Furthermore, we showed that both anti-FIM and antiFHA sera and mAbs induce $B$. pertussis aggregation and bacterial cell death, reducing bacterial growth. Inhibition of $B$. pertussis adhesion to A549 cells by antisera or mAbs also correlated with reduced production of inflammatory cytokines and increased A549 survival.

In summary, anti-FHA and anti-FIM antibodies were capable of sequestering $B$. pertussis in aggregates to kill the bacteria and prevent A549 cell inflammation, supporting the importance of humoral responses as a defense mechanism against $B$. pertussis.

\section{MATERIAL AND METHODS}

\section{Reagents}

Rabbit anti-human phospho p65 NF- $\kappa B$, PathScan ${ }^{\circledR}$ Signaling Nodes Multi-Target Sandwich ELISA, PathScan ${ }^{\circledR}$ signaling NF- $\kappa$ B ELISA, and PathScan ${ }^{\circledR}$ Sandwich ELISA Lysis Buffer were obtained from Cell Signaling Technology (Pickering, ON, Canada). Cy3-conjugated goat anti-mouse IgG was purchased from Invitrogen Canada (Burlington, ON, Canada). Bordet-Gengou blood agar plates were from BD Biosciences (Mississauga, ON, Canada). Syto9 and Propidium Iodide were from ThermoFisher (Waltham, MA). Wild-type $B$. pertussis (Tohama I) was propagated at Sanofi Pasteur (Toronto, ON, Canada). Human lung carcinoma epithelial A549 cell line was obtained from ATCC (Old Town Manassas, VA). For the cytokine detection (Eotaxin, Eotaxin-3, IL-10, IL-12/IL-23p40, IL-12p70, IL-13, IL-15, IL-16, IL-17A, IL-1 $\alpha$, IL-1 $\beta$, IL-2, IL-4, IL-5, IL-6, IL-7, IL-8, IL-8 (HA), IL-21, IL-22, IL-23, IL-27, IL31, IP-10, MCP-1, MCP-4, MDC, MIP- $1 \alpha$, MIP-1 $\beta$, MIP-3 $\alpha$, TARC, TNF- $\alpha$, TNF- $\beta$, VEGF-A, GM-CSF, and IFN- $\gamma$ ), V-PLEX Human Cytokine 36-Plex Kit (K15089D-2) was used for screening and only 20 selected cytokines were further tested on 20-plex cytokines (Meso Scale Discovery, Rockville, MD).

\section{Murine Monoclonal Antibodies}

The monoclonal antibodies (mAbs) were generated through Hybridoma Technology by Envigo using purified in-house protein (against FIM, FHA, and PT) and are property of Sanofi Pasteur Limited. Anti-FHA mAbs (clones 2-14, 2-2, 1-7, 3-31, 3 $35,5-6,28-1,2-3,1-14,1-11,32-1$ and 28-1) and anti-FIM mAbs (clones 1-1, 1-3, 1-7, C10, G10 and 1-10), and anti-PT mAbs (clones LP12 and PS21C) were first screened for their ability to inhibit $B$. pertussis growth. Selected anti-FHA (2-14, 1-7, 28-1, 11-1, 1-9, and 32-1) and anti-FIM (1-1, G10, 1-7, 1-10, and C10) $\mathrm{mAbs}$ were further investigated for co-culture studies using $B$. pertussis and A549 cells. Only anti-FHA (28-1) and FIM (1-10) were using for adhesion inhibition assay.

\section{Guinea Pig Immunization With aP Antigens}

Twenty-five guinea pigs were immunized with single aP antigens (FIM or FHA) adjuvanted with $\mathrm{AlPO}_{4}$ or aP vaccine (which contains FIM, FHA, PT, PRN). Sera from guinea pigs immunized with diphtheria antigen were used as control in this study. All animals were housed in pathogen-free research vivarium at Sanofi Pasteur animal care facility. All procedures were compliant with Guidelines of the Canadian Council on Animal Care and recommendations of Helsinki protocol.

\section{A549 Cell and Bordetella pertussis Culture}

Bordetella pertussis Tohama I strain was obtained from the collection of Microbiology Unit, Sanofi Pasteur, Canada. Human alveolar basal epithelial cells (A549) were cultured in Dulbecco's Modified Eagle Medium (DMEM; Gibco, Gaithersburg, MD) supplemented with $10 \%$ fetal bovine serum (HyClone/Cytiva, Vancouver, British Columbia, Canada) and incubated at $37^{\circ} \mathrm{C}$ and $5 \% \mathrm{CO}_{2}$. On the day of the assay, frozen 
vials of $B$. pertussis were thawed at room temperature. The bacteria were harvested by centrifugation at 10,000 $\mathrm{x} g$ for $3 \mathrm{~min}$ followed by re-suspension of the pellet in $1 \mathrm{ml}$ of DPBS (Gibco), twice. For infection, $5 \times 10^{5}$ A549 cells were co-cultured with $5.55 \times 10^{7}$ B. pertussis at multiplicity of infection (MOI) of 111 . Bacteria were first incubated with polyclonal sera or mAbs for $1 \mathrm{~h}$ before introduction to A549 cells. A549 cells and B. pertussis coculture was performed in 12-well plates. A549 cell morphological changes and bacterial aggregations were observed after $48 \mathrm{~h}$ of coculture by a bright-field compound microscope (Nikon) as previously described (22). After $72 \mathrm{~h}$ of incubation, the cell culture media from the coculture plate was collected and centrifuged at 13,000 $\mathrm{x}$ g for $3 \mathrm{~min}$. The cleared supernatants were stored at $-20^{\circ} \mathrm{C}$ for cytokine and chemokine detections. The remaining pellet was resuspended in $1 \mathrm{ml}$ of F11-medium. Bacterial concentration was determined by a spectrophotometer (SpectraMax, Molecular Devices, San Jose, CA) at $\mathrm{OD}_{620 \mathrm{~nm}}$. All bacterial clumps were broken up by vortexing and pipetting repeatedly. Dispersion of clumps was confirmed using light microcopy. Afterwards, bacteria were diluted $10^{7}$-fold by serial dilutions and $0.5 \mathrm{ml}$ was plated on Bordet-Gengou agar and colonies counted after $5-7$ days of culture at $37^{\circ} \mathrm{C}$.

For adhesion assay, co-culture suspension was cultured on a sterile cover slip, then fixed with $4 \%$ paraformaldehyde (PFA) before immunofluorescence staining. To test the direct bactericidal effects of anti-pertussis antibodies, B. pertussis was grown in the presence of mAbs or anti-sera, in the absence of A549 cells, as mentioned above.

\section{A549 Cell Lysate Preparation for NF- $\mathrm{kB}$ Detection}

After $48 \mathrm{~h}$ of co-culture of A549 cells with B. pertussis, culture supernatants were removed, and adherent cells washed three times with $1 \mathrm{ml}$ of cold DPBS. Other controls including a positive control (LPS) and a negative control (resting cells) were also included. A549 cells were trypsinized and incubated with $0.5 \mathrm{ml}$ ice-cold 1X cell lysis buffer with a protease inhibitor for $15 \mathrm{~min}$ on ice. Cytoplasmic fractions of cell lysates were obtained by centrifugation at $10,000 \mathrm{xg}$ for $5 \mathrm{~min}$ at $4^{\circ} \mathrm{C}$. The supernatant of cell lysates was collected for NF- $\kappa B$ detection and stored at $-20^{\circ} \mathrm{C}$ as single-use aliquots.

\section{Cytoplasmic Phospho-p65 NF-кB Detection by ELISA}

The cytoplasmic phospho- NF-אB p65 (Ser536) levels were determined as per manufacturer's instructions (Cell Signaling Technology ${ }^{\circledR}$ ). Briefly, $100 \mu \mathrm{l}$ of each undiluted A549 cell lysate was added to the pre-coated plate and incubated overnight at $4^{\circ} \mathrm{C}$. After four washes of the plate with $200 \mu \mathrm{l}$ of $1 \mathrm{X}$ wash buffer, $100 \mu \mathrm{l}$ of reconstituted detection antibody was added to each well. The plate was incubated at $37^{\circ} \mathrm{C}$ for $1 \mathrm{~h}$. After washing, 100 $\mu \mathrm{l}$ of reconstituted HRP-linked secondary antibody was added to each well and the plates were incubated for $30 \mathrm{~min}$ at $37^{\circ} \mathrm{C}$. After another four washes, $100 \mu \mathrm{l}$ of TMB substrate was added to each well and the plates incubated at $37^{\circ} \mathrm{C}$ for $30 \mathrm{~min}$. Reactions were stopped with $100 \mu \mathrm{l} /$ well of maleic acid and the plates were read within $30 \mathrm{~min}$ by a spectrophotometer (SpectraMax) at $450 \mathrm{~nm}$.

\section{Detection of Cytokines and Chemokines Using Meso Scale Discovery Technology}

The assay for detection of cytokines, chemokines and other inflammatory mediators was performed as per the manufacturer's recommendation (MSD). Briefly, $50 \mu \mathrm{l}$ cell supernatant from $72 \mathrm{~h}$ cell culture media and standards were added to MSD 20-plex precoated plates. The plate was incubated at room temperature for $2 \mathrm{~h}$ and washed 3 times with wash buffer to remove the unbound analytes. Twenty-five microliters of diluted SULFO-TAGconjugated detection antibodies were added, and the plate was incubated for $2 \mathrm{~h}$ at room temperature. The plate was washed tree times, MSD "Read" buffer was added, and the plate was analyzed with an MSD instrument.

\section{Immunofluorescence Staining, Microscopy, ImageStream, and Flow Cytometry}

After 48 h of co-culture of B. pertussis and anti-FIM or anti-FHA mAbs with A549 cells, the supernatant was removed, and adherent cells washed 3 times with $1 \mathrm{ml}$ of DPBS. After blocking (4\% bovine serum albumin (BSA) in $1 \mathrm{X}$ Tris-buffered saline (TBS)-Tween), adherent bacteria on A549 cells were stained with secondary goat anti-mouse Cy3-conjugated antibody to reveal the presence of anti-pertussis mAbs [control (Ctrl) anti-PRN (3-5), anti-FHA (28-1) anti-FIM (1-10)] on the bacteria. After washing, the cells were mounted with ProLong Gold Antifade Mountant (ThermoFisher) containing DAPI and observed by a fluorescent microscope (Zeiss Axio; Zeiss; Oberkochen, Germany).

Bacterial viability assessment was performed by staining a fraction of the suspension of mAb-treated bacteria collected after $48 \mathrm{~h}$ culture. Bacteria were stained with $0.0125 \mu \mathrm{M}$ of Syto9 and $7.5 \mu \mathrm{M}$ of propidium iodide (PI) for $15 \mathrm{~min}$. Fluorescently stained bacteria were analyzed with Flow cytometry (BD FACSCalibur, BD).

To investigate the correlation between bacterial death and aggregation, Syto9 or Propidium Iodide PI stained B. pertussis were analyzed on the Amnis ${ }^{\circledR}$ ImageStream ${ }^{\circledR} \mathrm{X}$ Mk II Imaging Flow Cytometer. Both dyes were excited with the $488 \mathrm{~nm}$ laser, and the Syto 9 or PI fluorescence was detected with either a $505-560 \mathrm{~nm}$ or a 595-642 $\mathrm{nm}$ band pass filter respectively at $60 \mathrm{x}$ magnification. The brightfield image and side scatter were also captured. Samples were analyzed using IDEAS software version 6.2.187.0. To analyze the bacteria, in focus events were gated using "Gradient RMS" values over 40, followed by Syto 9 and PI positive events with fluorescence intensities greater than 500 and 1000, respectively. Aggregated dead bacteria were defined as having large size via the brightfield image and/or having high mean pixel intensity of Syto9 fluorescence.

\section{Statistical Analysis}

Data are shown as the mean \pm SEM. Statistical comparisons were made using an unpaired Student's t-test. Immunofluorescence analysis was performed using Image J software (National Institutes of Health). $\mathrm{P}$ values $\leq 0.05$ were considered statistically significant. 


\section{RESULTS}

\section{Interactions Between A549 Epithelial Cells and Bordetella pertussis Enhance Bacterial Growth Which Is Inhibited by Specific mAbs Against FIM and FHA}

During pertussis infection, common adhesion molecules (FIM and FHA) bind to their cognate ligands on epithelial cells, allowing the bacteria to escape the immune system and colonize the respiratory tract. In a co-culture system, we observed that bacterial growth was increased in the presence of A549 epithelial cells in a dose-dependent manner, compared to B. pertussis cultured alone in complete DMEM media (Figure 1A). Through this model, we were able to evaluate the inhibition of bacterial proliferation in the presence of guinea pig polyclonal sera and mouse monoclonal antibodies. The ability of antisera (polyclonal sera against either purified FIM or FHA antigen, or from immunization with aP vaccine) to reduce bacterial growth was first explored. To enumerate live bacteria, B. pertussis in the supernatants from $72 \mathrm{~h}$ co-cultures were plated on BordetGengou agar. The results showed that the guinea pig antisera raised against aP vaccine, FHA or FIM purified antigens, significantly reduced the bacterial growth compared to naïve control sera or antisera raised against PT (Figure 1B). The reduction of bacterial growth in the presence of antibodies could also be achieved using anti-FHA (clones 28-1, 1-11, and 1-9) and anti-FIM (clones G10, 1-7, and 1-10) mAbs on bacterial growth (Figure 1C). The data suggested inhibition of $B$. pertussis growth by anti-FIM and anti-FHA antibodies through a mechanism that involves prevention of $B$. pertussis interactions with A549 cells.

\section{Polyclonal Antisera and Specific mAbs Induce Bordetella pertussis Aggregation and Improve A549 Cell Survival}

To explore the potential mechanism involved in bacterial growth inhibition, aggregation of $B$. pertussis in the presence of antibodies were investigated. Resting A549 cells reached 98\%$100 \%$ confluency after $72 \mathrm{~h}$ of culture and formed a healthy monolayer. Infection of A549 cells by B. pertussis in the presence of naïve sera led to epithelial cell death, clump or cluster formation, and reduction in numbers of A549 cells (Figure 2A). Interestingly, antibodies against aP vaccine, anti-FHA, or anti-FIM sera induced bacterial aggregation after $48 \mathrm{~h}$ of incubation. We have shown that anti-PT sera induced weak and reversable aggregation in only half of the tested samples (data not shown). The anti-PT sera was used in this assay to compare the results with anti-FIM and anti-FHA sera. AntiFHA mAbs (seven out of 12 clones) and all anti-FIM mAbs (six out of six clones) induced $B$. pertussis aggregation (data not shown).

The role of B. pertussis adhesins in binding to A549 cells was also evaluated in this study. After $48 \mathrm{~h}$ incubation of mAbtreated B. pertussis with A549 cells, an adhesion assay was performed. To visualize the interaction of bacteria with A549 cells, anti-FIM (1-10) and anti-FHA (28-1) mAbs bound to the
B. pertussis surface were stained with Cy3-conjugated goat antimouse antibodies. Notably anti-FHA and anti-FIM reduced bacteria adhesion to A549 cells (Figure 2B) as revealed by fluorescence microscopy. Anti-PRN mAb used as the control as we have previously shown that this $\mathrm{mAb}$ does not induce aggregation. In addition to microscopy observations, we tested the ability of aggregation-inducing antibodies to prevent bacterial adhesion to A549 cells following $48 \mathrm{~h}$ of coculture. After removing unbound $B$. pertussis through washing, aP vaccine, anti-FHA, and anti-FIM sera, which induced bacteria aggregation, were shown to significantly reduce CFU count of adherent bacteria (Figure 2C).

\section{Aggregation by Anti-Pertussis Antibodies Induces Direct Bactericidal Effect on Bordetella pertussis, and Restores A549 Cell Survival}

A flow cytometry-based approach was developed and used to further investigate the bactericidal properties of anti-pertussis $\mathrm{mAb}$. For this purpose, anti-pertussis antibody-treated bacteria were cultured in DMEM for $48 \mathrm{~h}$ without A549 cells. Staining of live/dead bacteria indicated that the bacterial death occurs in the presence of anti-FIM (1-10) or anti-FHA (28-1) antibodies alone (Figure 3A). Complementary analysis using a flow imaging system (ImageStream) showed a similar pattern to the traditional flow cytometry (Figures 3A, B). In addition, the ImageStream system was used to further analyze aggregation within the dead bacteria population using high magnification (60X). In this study, aggregation was defined as events with large size by brightfield and/or with high mean Syto 9 fluorescence, an indication that the bacteria are tightly compacted (Figure 3B, center plot). Treatment of $B$. pertussis with FIM (1-10) or FHA (28-1) antibodies resulted in the formation of large aggregates in the dead bacteria population as compare to the control. The potential of anti-pertussis antibodies to prevent attachment or adhesion (which is a key step for infection) to epithelial cells or to kill $B$. pertussis in the aggregates may represent an interesting mechanism of action of mAbs on the pathogen.

\section{Antibodies That Mediate B. pertussis Aggregation Restores A549 Cell Survival and Alters Cytokine/Chemokine Secretions}

To evaluate the effects of anti-pertussis antibodies on A549 cells, a neutral red viability assay was conducted in order to assess cell survival in the presence of $B$. pertussis. Neither naïve sera or antidiphtheria toxin (DIP) control sera protected A549 cells from B. pertussis, induced A549 cell cluster formation and cell death (Figure 4A). In contrast, antisera containing antibodies against aP vaccine, FHA, or FIM antigens significantly improved A549 cell survival (Figure 4A). This was also observed in the presence of three anti-FHA mAbs (clones 2-14, 1-7, and 28-1) and three anti-FIM mAbs (clones 1-1, 1-3,1-7, and 1-10), which significantly prevented A549 cell death.

To evaluate the impact of the inhibition of $B$. pertussis interaction with A549 cells by anti-FIM and anti-FHA sera, 
A Bacterial growth in co-culture with A549 cells

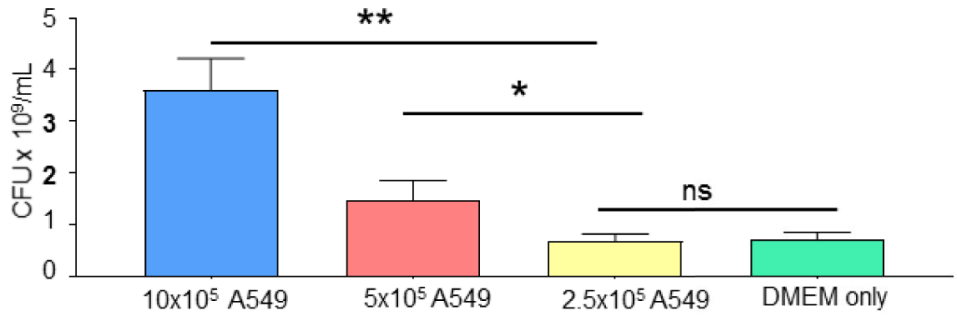

B Inhibition of bacterial growth by immunized guinea pig sera

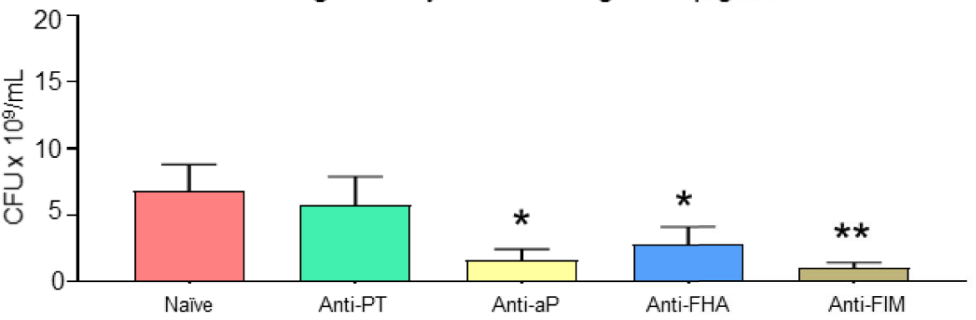

C Inhibition of bacterial growth by mouse monoclonal antibodies
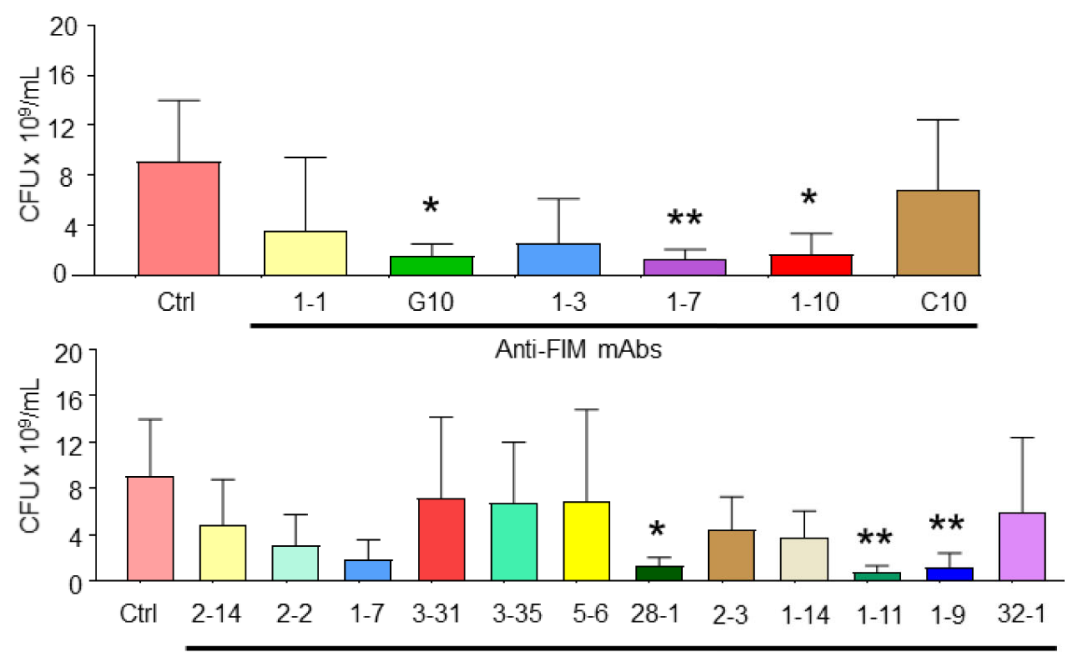

Anti-FHA mAbs

FIGURE 1 | Bordetella pertussis interactions with A549 epithelial cells are essential for bacterial growth which is inhibited by specific mAbs. (A) B. pertussis growth significantly increased in a dose dependent manner in presence of 1 and 0.5 million of A549 cells. After $72 \mathrm{~h}$ co-culture of $B$. pertussis with A549 cells, extracellular bacterial concentration in the medium was determined. (B, C) Enumeration of live bacteria on Bordet-Gengou blood agar showed significant inhibition of $B$. pertussis growth by anti-sera (B) compared to naive control sera. Mouse mAb targeting FIM (G10, 1-7, and 1-10) and FHA (28-1, 1-11, and 1-9) significantly inhibited bacterial growth compared to anti-RSV control mAb (C). Unpaired Student's $t$-test. Mean \pm SEM. ${ }^{*} p<0.05$, and ${ }^{* *} p<0.01, \mathrm{n} \geq 4$ experiments. ns, non significant.

pro-inflammatory signaling and cytokine secretions were investigated in the co-cultured A549 cells. Specifically, this work leveraged $\mathrm{NF}-\kappa \mathrm{B}$, a downstream target mediating inflammation which has previously been shown to be activated upon by binding of $B$. pertussis adhesins to Toll-like receptors TLR2 and TLR4 (23). Translocation of NF- $\kappa B$ from the cytoplasm to the nucleus initiates transcription of various cytokine genes. In this study, the level of cytoplasmic phosphor-p65 of NF- $\kappa \mathrm{B}$ was enhanced in the presence of antiFIM or anti-FHA sera, suggesting less translocation of NF- $\kappa \mathrm{B}$ to the host cell nucleus and reduced transcription of pro- inflammatory genes. As expected, in the presence of anti-PT sera, the level of cytoplasmic phosphor-p65 was similar to control sera (Figure 5A).

Activation of NF- $\mathrm{KB}$ is a prerequisite for pro-inflammatory cytokine secretions; therefore, the cytokine profile of A549 infected cells in the presence of different antisera was further investigated. Thirty-six cytokines were first screened by a multiplex panel and then narrowed down to 20 cytokines and chemokines of interest. The level of several pro-inflammatory cytokines (IFN $\gamma$, IL-1 $\beta$, IL-6, and IL-8) and chemokines (MIP$1 \alpha$ chemokine (C-C motif) ligand (CCL3), MIP-1 $\beta$ (CCL4), 
A Immunized guinea pig anti-sera

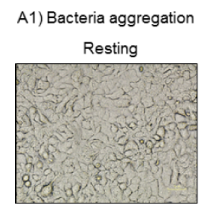

Anti-aP sera
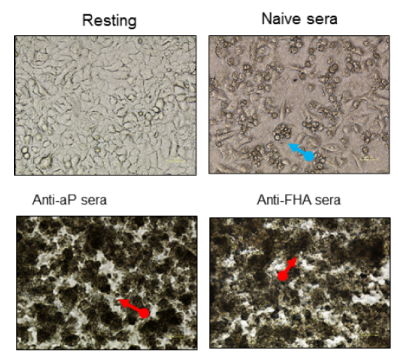

Anti-FHA sera

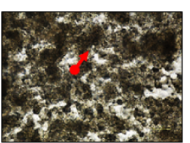

Mouse monoclonal antibodies

A2) Bacteria aggregation

$$
\text { Ctrl mAb }
$$

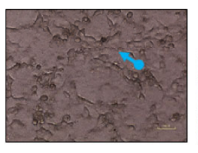

Anti-FHA mAb(1-11)

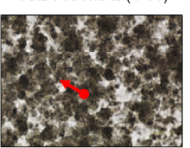

B Inhibition of bacteria adhesion to A549 cells Inhibition of adhesion by immunofluorescence

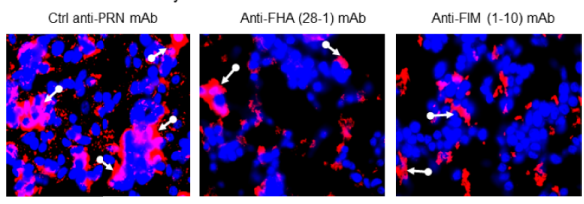

Anti-FIM sera
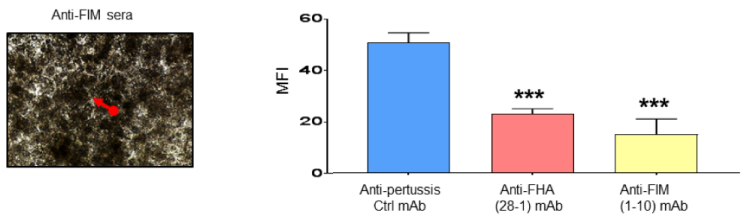

C Inhibition of adhesion by CFU enumeration

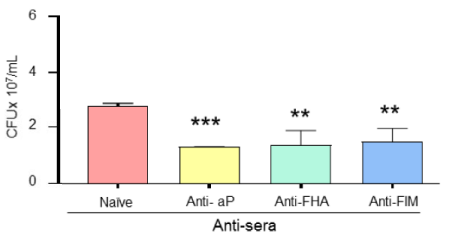

FIGURE 2 | Antibodies against B. pertussis key adhesins induced bacterial aggregation and inhibit adhesion. (A) Naïve control sera did not protect infected A549 cells which exhibited cluster formation and cell death (blue arrows) compared to confluent resting cells. Naiive sera did not induce bacteria aggregation (A1). Antisera containing antibodies against aP vaccine or single immunogens, FHA, and FIM, induced bacterial aggregation (red arrows) in $100 \%$ of tested sera as certain antiFHA and anti-FIM mAbs induced bacterial aggregation (A2). (B) Specific anti-pertussis antibodies inhibit bacterial adhesion. After $48 \mathrm{~h}$ of co-culture, anti-FIM and anti-FHA mAb significantly inhibited bacteria adhesion compared to control anti-PRN mAb (white arrow) to A549 cells which was confirmed using bacteria enumeration on Bordet-Gengou plate (C). Mean \pm SEM. ${ }^{\star \star *} p<0.001$ and ${ }^{\star \star} p<0.01, \mathrm{n} \geq 3$ experiments.

A Bactericidal effect of mAbs by flow cytometry
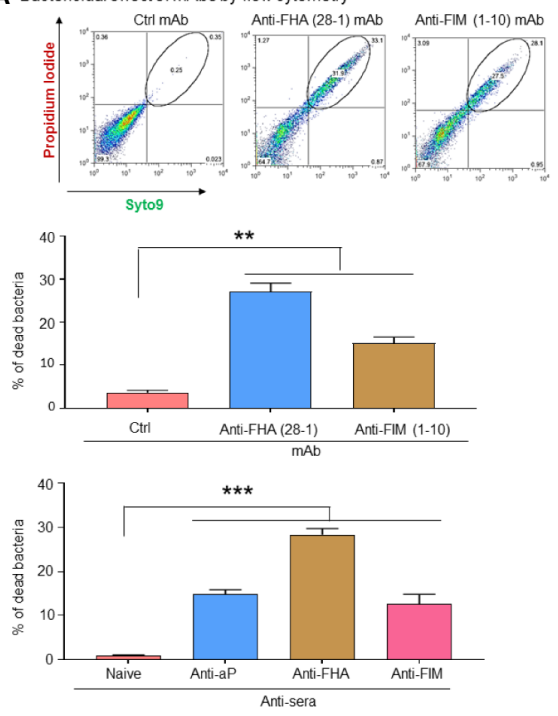

B Aggregation and bacteria death Live/dead bacteria $\quad \begin{aligned} & \text { Aggregation of } \\ & \text { dead population }\end{aligned}$

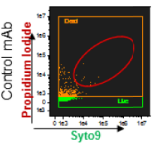
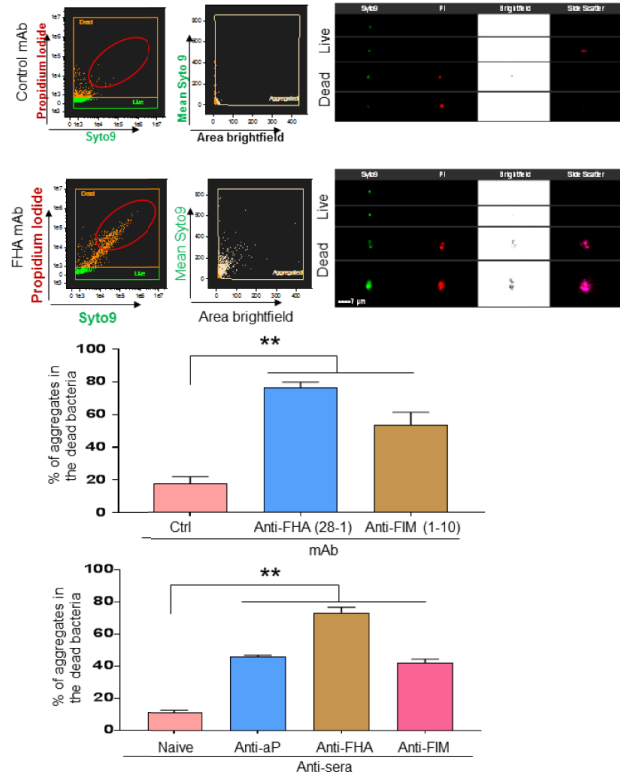

FIGURE 3 | B. pertussis sequestration by aggregation inducing antibodies mediated direct bactericidal effects. B. pertussis was incubated for 48 h with antipertussis antibodies without A549 cells to test the direct bactericidal effect of anti-FIM and anti-FHA antibodies. Flow cytometry-based analysis of bacteria death shows that anti-pertussis antibodies against FIM and FHA exhibit direct bactericidal effect (A). Furthermore, analysis by imaging flow cytometry showed that majority of dead bacteria are within the aggregates (B). Unpaired Student's $t$-test. Mean \pm SEM. ${ }^{\star \star} p<0.01$ and ${ }^{\star \star \star} p<0.001, n \geq 3$ experiments. 


\section{A A549 cell survival in presence of anti-sera}

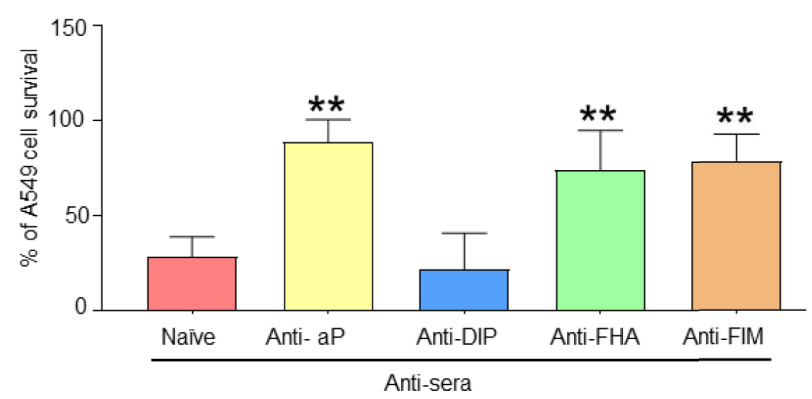

B A549 cell survival in presence mAbs
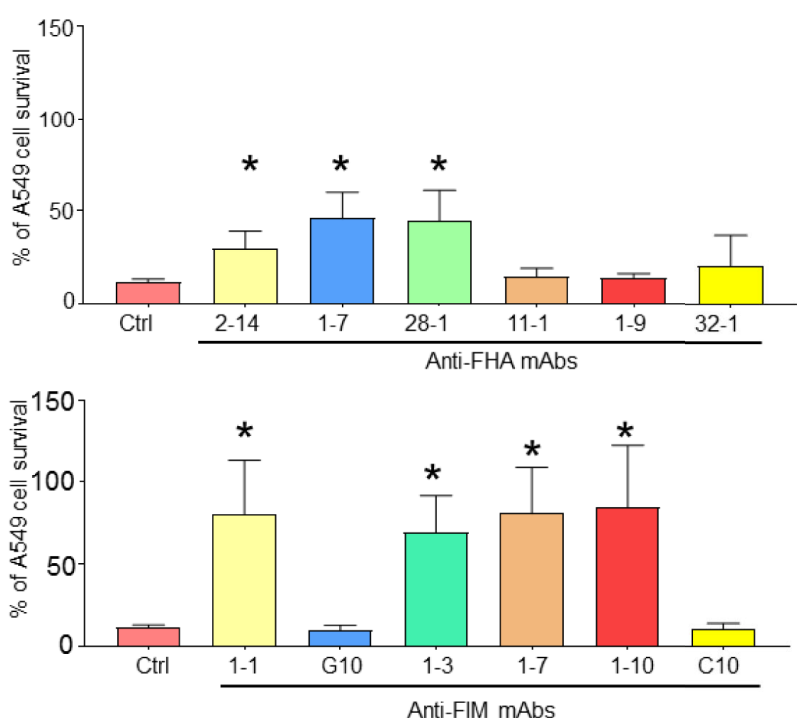

FIGURE 4 | Bacteria aggregation and inhibition of adhesion improve A549 cell survival. After $72 \mathrm{~h}$ of infection, remaining adherent A549 cells were stained with Neutral red for cell viability/proliferation. Anti-FIM and anti-FHA anti-sera, which induced B. pertussis aggregation have a beneficial impact on A549 cell survival (A). This observation was reproducible by certain specific anti-FIM and anti-FHA mAbs (B). Unpaired Student's t-test. Mean \pm SEM. ${ }^{*} \mathrm{p}<0.05$ and ${ }^{* *} p<0.01 . n \geq 3$ experiments.

MCP-4 (CCL2), and Eotaxin-3 (CCL26) were significantly reduced in the presence of anti-FHA or anti-FIM sera (Figure 5B). These results indicate that $B$. pertussis aggregation prevents interaction between bacteria and A549 cells, thus improving epithelial cell survival and preventing proinflammatory response.

\section{DISCUSSION}

Pertussis continues to be a major public health concern especially in infants despite high coverage of vaccines (7). Recent findings have shown the urgent need for pertussis vaccines, particularly in the prenatal stage of life, to reduce severe pertussis in newborns (24). For this particular reason, the advisory committee on immunization practices of Centers for Disease Control and
Prevention recommended immunization of pregnant women with acellular pertussis vaccine to protect their infants against pertussis via transplacental antibody transfer $(8,25)$. Antibodies play a major role in the protection of newborns from severe pertussis, however the mechanisms by which anti-pertussis antibodies exert direct bactericidal effects to control epithelial infection has remained elusive (26).

From this study, one possible mechanism of action for antibodies against certain $B$. pertussis adhesins is the induction of bacterial aggregation to sequester and kill the bacteria. We have performed multiple assays to obtain mechanistic insight into the bactericidal properties of anti-pertussis antibodies and their protective effects on A549 cells. We found that in the presence of B. pertussis, A549 epithelial cells release biological factors supporting bacterial growth. By sequestering $B$. pertussis into aggregates, anti-FIM and anti-FHA antibodies inhibit bacteria interactions with A549 cells, a prerequisite for enhanced bacterial growth in our co-culture system.

The observation that $B$. pertussis experiences more rapid growth in the presence of A549 cells prompted us to establish a cellular model for proper investigation on the bactericidal properties of vaccine-induced antibodies. We hypothesize that during infection, interaction of $B$. pertussis with epithelial cells mediate release of variety of factors from infected A549 cells which facilitate bacteria growth through a direct pathogen-host cell interaction. This is in agreement with a previous study showing that $B$. pertussis interactions with respiratory epithelial ciliated cells led to five-fold increase in bacterial density within $24 \mathrm{~h}$ (27). When B. pertussis interacts with epithelial cells through its major adhesins, it may induce signaling for bacterial replication. Using filtered media from the co-culture system, enhanced bacterial growth was observed; albeit further study is required to identify and investigate any possible secreted biological factor(s) and/or property of this 'conditioned' A549 cell media that may impact $B$. pertussis growth. Some factors such as $\mathrm{MgSO}_{4}$ and glutamate have been previously reported to affect $B$. pertussis growth $(28,29)$. For instance, conditions that restrict the availability of glutamate, a key essential amino acid, have been showed to reduce expression of virulence factors and adhesins, and entering stationary phase of $B$. pertussis. Since $B$. pertussis does not utilize sugars, but rather rely on glutamate, deprivation of this essential nutrient has been shown to induce autoaggregation (28). The fact that FHA and FIM are highly expressed on the pathogen at all stages of bacterial growth makes these antigens effectively targetable by antibodies. In fact, when B. pertussis is co-cultured with A549 in the presence of anti-FHA and anti-FIM mAbs or polyclonal sera, inhibition of bacterial adhesion/interactions prevented biological factor release. Inhibition of bacteria interactions with A549 cells by anti-FHA and FIM antibodies could explain the reduction of $B$. pertussis growth, however this does not occur without aggregation which sequesters the pathogen.

To increase pertussis vaccine efficacy, antigens that induce generation of bactericidal antibodies have been highly recommended. Of particular interest, engineering vaccines that promote antibodies with opsonophagocytic and/or bactericidal 
A Phospho-NF-kB p65

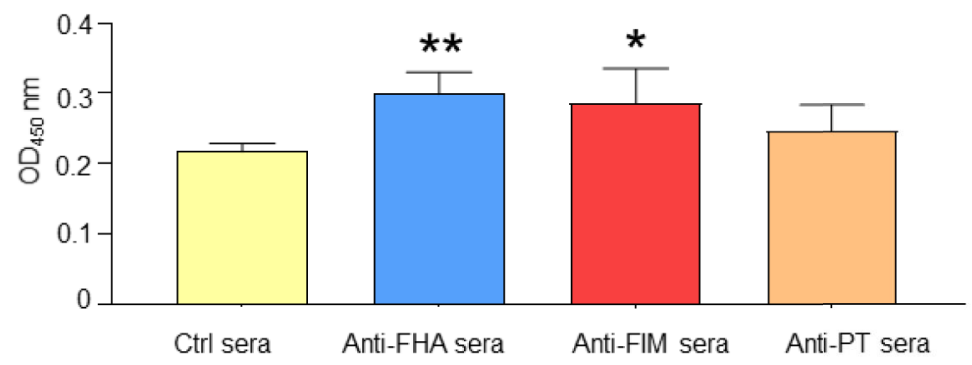

B pro-inflammatory and chemokine release
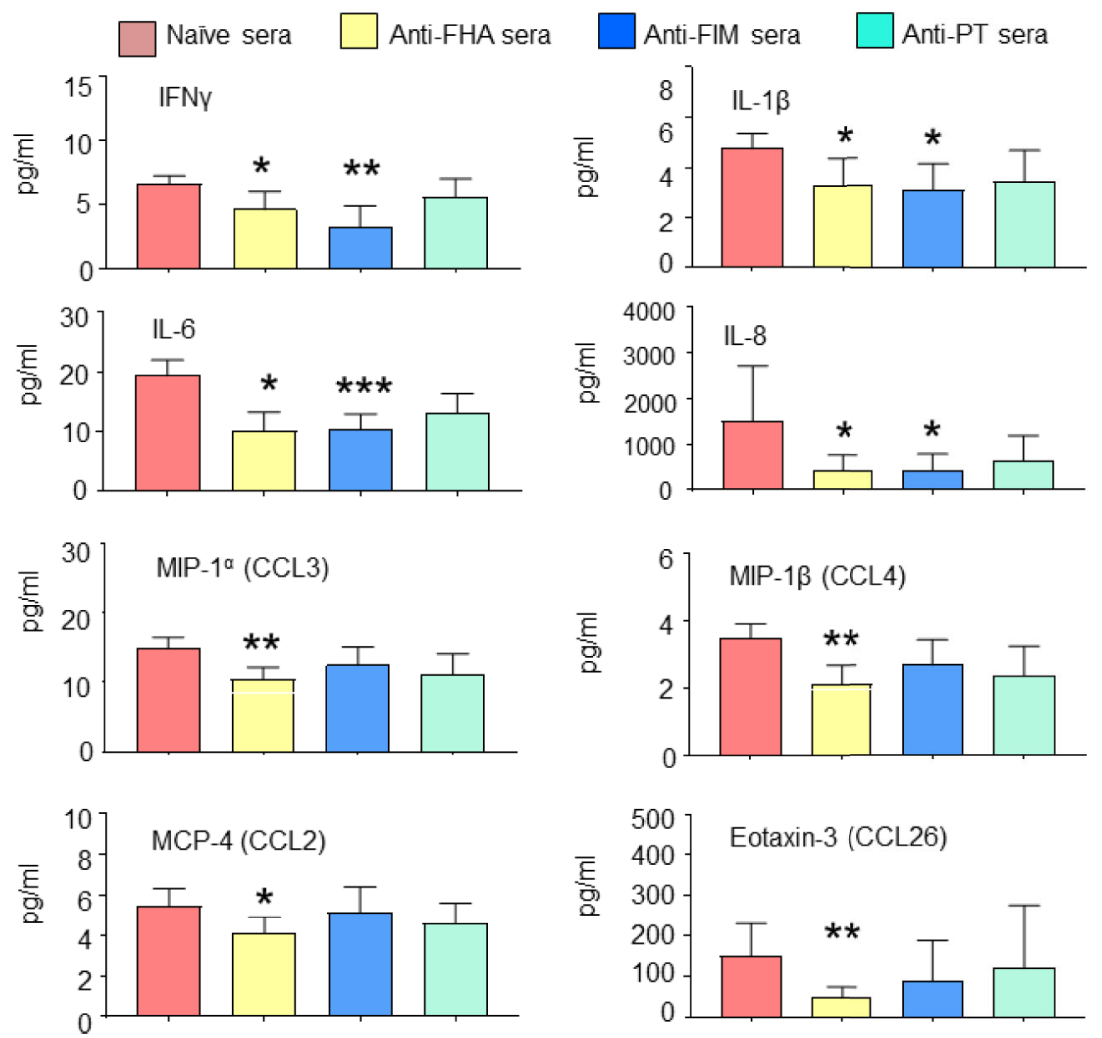

FIGURE 5 | Antibodies mediating B. pertussis aggregation reduced A549 pro-inflammatory signaling. (A) Anti-FHA and anti-FIM polyclonal sera reduced NF- $\mathrm{BB}$ activation and $\mathrm{A} 549$ cell inflammatory response. Cytoplasmic NF- $\mathrm{KB}$ levels remains higher in presence of anti-FIM and anti-FHA sera compared to control sera. (B) Complementary analysis of pro-inflammatory cytokines and chemokines released in the culture supernatant in response to $B$. pertussis infection were significantly reduced by anti-FIM and anti-FHA compared to naive sera. Unpaired Student's $t$-test. Mean \pm SEM. ${ }^{*} p<0.05,{ }^{* *} p<0.01,{ }^{* \star *} p<0.001 \mathrm{n} \geq 3$ experiments.

activity may improve efficacy (30). There are discrepancies between the existing studies investigating opsonophagocytic and bactericidal activities induced by aP vaccines $(31,32)$. In a study by Lesne and colleagues, contribution of PRN to generate complement-mediated bactericidal antibodies has been reported while anti-FIM, anti-FHA, and anti-PT antibodies against pertussis were not involved (31). However, some controversies remain as other studies showed that levels of anti-PT and FIM antibodies directly correlated with the protection in humans $(32,33)$. In our study all the animals immunized with FHA or FIM immunogens developed antibodies that induce $B$. pertussis aggregation. Aggregation can be visualized after $24 \mathrm{~h}$ and aggregates grew over the time. It is a dynamic process in which the aggregates become larger over time after $48 \mathrm{~h}$. Bacterial death within the aggregates was demonstrated by examining at the population of aggregated $B$. pertussis stained by Syto 9 and propidium iodide live/dead markers. Aggregation blocks pathogen-host cell interactions, which may in turn disrupt some factors that are needed for bacterial proliferation (e.g. host signals that trigger 
bacterial proliferation or growth, or factors that may be derived from other bacterial interactions). It is also known that $B$. pertussis is very sensitive to temperature and nutrients (e.g. $\mathrm{MgSO}_{4}$ and glutamate) for expression of key virulent factors. It is possible that the contact inhibition between neighboring bacteria may trigger a decline in cell cycle or even more cell death. Taken together, microbial pathogens must sense local conditions, including space to grow, nutrient availability, and adjust their growth state accordingly to survive in a host environment. Therefore, anti-FHA and anti-FIM antibodies may block the biosynthesis of necessary virulence factors associated with cell division.

In agreement with bacterial aggregation and growth inhibition by anti-FIM and anti-FHA mAbs, survival of A549 cells was significantly improved. Stimulation of inflammation was also prevented as key anti-inflammatory cytokines and chemokines were reduced in the presence of anti-FIM and anti-FHA antibodies (Scheme 1). By sequestering B. pertussis in aggregates, anti-pertussis antibodies can reduce bacterial growth and prevent A549 cell inflammation, demonstrating their potential for development of in vitro potency assays, supporting initiatives to reduce animal-based testing.

\section{REFERENCES}

1. Klein NP, Bartlett J, Rowhani-Rahbar A, Fireman B, Baxter R. Waning protection after fifth dose of acellular pertussis vaccine in children. $N$ Engl J Med (2012) 367:1012-9. doi: 10.1056/NEJMoa1200850

2. Koepke R, Eickhoff JC, Ayele RA, Petit AB, Schauer SL, Hopfensperger DJ, et al. Estimating the effectiveness of tetanus-diphtheria-acellular pertussis vaccine (Tdap) for preventing pertussis: evidence of rapidly waning immunity and difference in effectiveness by Tdap brand. J Infect Dis (2014) 210:942-53. doi: 10.1093/infdis/jiu322

3. Mills KH, Ross PJ, Allen AC, Wilk MM. Do we need a new vaccine to control the re-emergence of pertussis? Trends Microbiol (2014) 22:49-52. doi: 10.1016/j.tim.2013.11.007

4. Godfroid F, Denoel P, Poolman J. Are vaccination programs and isolate polymorphism linked to pertussis re-emergence? Expert Rev Vaccines (2005) 4:757-78. doi: 10.1586/14760584.4.5.757

5. Williams MM, Sen K, Weigand MR, Skoff TH, Cunningham VA, Halse TA, et al. Bordetella pertussis Strain Lacking Pertactin and Pertussis Toxin. Emerg Infect Dis (2016) 22:319-22. doi: 10.3201/eid2202.151332

6. Skoff TH, Faulkner AE, Liang JL, Barnes M, Kudish K, Thomas E, et al. Pertussis Infections among Pregnant Women in the United States, 2012-2017. Clin Infect Dis (2020) ciaa1112. doi: 10.1093/cid/ciaa1112

7. Gilley M, Goldman RD. Protecting infants from pertussis. Can Fam Physician (2014) 60:138-40.

8. Esposito S. Prevention of pertussis: from clinical trials to Real World Evidence. J Prev Med Hyg (2018) 59:E177-86. doi: 10.15167/2421-4248/ jpmh2018.59.3.1041

9. Tanaka M, Vitek CR, Pascual FB, Bisgard KM, Tate JE, Murphy TV. Trends in pertussis among infants in the United States, 1980-1999. JAMA (2003) 290:2968-75. doi: 10.1001/jama.290.22.2968

10. Voysey M, Kelly DF, Fanshawe TR, Sadarangani M, O’Brien KL, Perera R, et al. The Influence of Maternally Derived Antibody and Infant Age at Vaccination on Infant Vaccine Responses : An Individual Participant Meta-analysis. JAMA Pediatr (2017) 171:637-46. doi: 10.1001/jamapediatrics.2017.0638

11. Barug D, Pronk I, van Houten MA, Versteegh FGA, Knol MJ, van de Kassteele J, et al. Maternal pertussis vaccination and its effects on the immune response of infants aged up to 12 months in the Netherlands: an open-label, parallel, randomised controlled trial. Lancet Infect Dis (2019) 19:392-401. doi: 10.1016/S1473-3099(18)30717-5

\section{DATA AVAILABILITY STATEMENT}

The raw data supporting the conclusions of this article will be made available by the authors, without undue reservation.

\section{AUTHOR CONTRIBUTIONS}

IY designed and performed all the experiments, analyzed and interpreted the data, and prepared and revised the manuscript. AM conducted several cell-based assays and prepared the data. LH and CC conducted flow cytometry experiments and prepared the manuscript. JS and BG provided guidance for study design and data interpretation. AA supervised the study, interpreted the data, and revised the manuscript. All authors contributed to the article and approved the submitted version.

\section{ACKNOWLEDGMENTS}

We thank Drs. Jason Szeto, Martina Ochs, Neil Blackburn, and Yuanqing Liu for critical review of the manuscript. We would also like to thank Mr. Jason Levere for preparation of B. pertussis strains.

12. Rice TF, Diavatopoulos DA, Smits GP, van Gageldonk PGM, Berbers GAM, van der Klis FR, et al. Antibody responses to Bordetella pertussis and other childhood vaccines in infants born to mothers who received pertussis vaccine in pregnancy - a prospective, observational cohort study from the United Kingdom. Clin Exp Immunol (2019) 197:1-10. doi: 10.1111/ cei. 13275

13. Bernstein HH, Rothstein EP, Reisinger KS, Blatter MM, Arbeter AM, Fontana $\mathrm{ME}$, et al. Comparison of a three-component acellular pertussis vaccine with a whole-cell pertussis vaccine in 15- through 20-month-old infants. Pediatrics (1994) 93:656-9.

14. Cowell JL, Zhang JM, Urisu A, Suzuki A, Steven AC, Liu T, et al. Purification and characterization of serotype 6 fimbriae from Bordetella pertussis and comparison of their properties with serotype 2 fimbriae. Infect Immun (1987) 55:916-22. doi: 10.1128/IAI.55.4.916-922.1987

15. Scheller EV, Melvin JA, Sheets AJ, Cotter PA. Cooperative roles for fimbria and filamentous hemagglutinin in Bordetella adherence and immune modulation. MBio (2015) 6:e00500-15. doi: 10.1128/mBio.00500-15

16. Cotter PA, Yuk MH, Mattoo S, Akerley BJ, Boschwitz J, Relman DA, et al. Filamentous hemagglutinin of Bordetella bronchiseptica is required for efficient establishment of tracheal colonization. Infect Immun (1998) 66:5921-9. doi: 10.1128/IAI.66.12.5921-5929.1998

17. Brennan MJ, Shahin RD. Pertussis antigens that abrogate bacterial adherence and elicit immunity. Am J Respir Crit Care Med (1996) 154:S145-9. doi: 10.1164/ajrccm/154.4_Pt_2.S145

18. Coutte L, Alonso S, Reveneau N, Willery E, Quatannens B, Locht C, et al. Role of adhesin release for mucosal colonization by a bacterial pathogen. J Exp Med (2003) 197:735-42. doi: 10.1084/jem.20021153

19. Inatsuka CS, Julio SM, Cotter PA. Bordetella filamentous hemagglutinin plays a critical role in immunomodulation, suggesting a mechanism for host specificity. Proc Natl Acad Sci USA (2005) 102:18578-83. doi: 10.1073/ pnas.0507910102

20. Tsang RSW, Shuel M, Cronin K, Deng S, Whyte K, Marchand-Austin A, et al. The evolving nature of Bordetella pertussis in Ontario, Canada, 2009-2017: strains with shifting genotypes and pertactin-deficiency. Can J Microbiol (2019) 65(11):823-30. doi: 10.1139/cjm-2019-0128

21. van den Berg BM, Beekhuizen H, Willems RJ, Mooi FR, van Furth R. Role of Bordetella pertussis virulence factors in adherence to epithelial cell lines derived from the human respiratory tract. Infect Immun (1999) 67:1056-62. doi: 10.1128/IAI.67.3.1056-1062.1999 
22. Shamova EV, Gorudko IV, Drozd ES, Chizhik SA, Martinovich GG, Cherenkevich SN, et al. Redox regulation of morphology, cell stiffness, and lectin-induced aggregation of human platelets. Eur Biophys J (2011) 40:195208. doi: 10.1007/s00249-010-0639-2

23. Asgarian-Omran H, Amirzargar AA, Zeerleder S, Mahdavi M, van Mierlo G, Solati S, et al. Interaction of Bordetella pertussis filamentous hemagglutinin with human TLR2: identification of the TLR2-binding domain. APMIS (2015) 123:156-62. doi: 10.1111/apm.12332

24. van der Maas NAT, Sanders EAM, Versteegh FGA, Baauw A, Westerhof A, de Melker HE. Pertussis hospitalizations among term and preterm infants: clinical course and vaccine effectiveness. BMC Infect Dis (2019) 19:919. doi: 10.1186/s12879-019-4563-5

25. C. Centers for Disease, and Prevention. Updated recommendations for use of tetanus toxoid, reduced diphtheria toxoid, and acellular pertussis vaccine (Tdap) in pregnant women-Advisory Committee on Immunization Practices (ACIP), 2012. MMWR Morb Mortal Wkly Rep (2013) 62:131-5. doi: 10.15585/mmwr.rr6702a1

26. Healy CM, Rench MA, Swaim LS, Smith EO, Sangi-Haghpeykar H, Mathis $\mathrm{MH}$, et al. Association Between Third-Trimester Tdap Immunization and Neonatal Pertussis Antibody Concentration. JAMA (2018) 320:1464-70. doi: 10.1001/jama.2018.14298

27. Soane MC, Jackson A, Maskell D, Allen A, Keig P, Dewar A, et al. Interaction of Bordetella pertussis with human respiratory mucosa in vitro. Respir Med (2000) 94:791-9. doi: 10.1053/rmed.2000.0823

28. Hanawa T, Kamachi K, Yonezawa H, Fukutomi T, Kawakami H, Kamiya S, et al. BvgAS Activation, and (p)ppGpp Regulate the Expression of the Bordetella pertussis Type 3 Secretion System. J Bacteriol (2016) 198:343-51. doi: 10.1128/JB.00596-15
29. Nakamura MM, Liew SY, Cummings CA, Brinig MM, Dieterich C, Relman DA. Growth phase- and nutrient limitation-associated transcript abundance regulation in Bordetella pertussis. Infect Immun (2006) 74:5537-48. doi: 10.1128/IAI.00781-06

30. Geurtsen J, Fae KC, van den Dobbelsteen GP. Importance of (antibodydependent) complement-mediated serum killing in protection against Bordetella pertussis. Expert Rev Vaccines (2014) 13:1229-40. doi: 10.1586/ 14760584.2014.944901

31. Lesne E, Cavell BE, Freire-Martin I, Persaud R, Alexander F, Taylor S, et al. Acellular Pertussis Vaccines Induce Anti-pertactin Bactericidal Antibodies Which Drives the Emergence of Pertactin-Negative Strains. Front Microbiol (2020) 11:2108. doi: 10.3389/fmicb.2020.02108

32. Cherry JD, Gornbein J, Heininger U, Stehr K. A search for serologic correlates of immunity to Bordetella pertussis cough illnesses. Vaccine (1998) 16:1901-6. doi: 10.1016/S0264-410X(98)00226-6

33. Storsaeter J, Hallander HO, Gustafsson L, Olin P. Levels of anti-pertussis antibodies related to protection after household exposure to Bordetella pertussis. Vaccine (1998) 16:1907-16. doi: 10.1016/S0264-410X(98)00227-8

Conflict of Interest: All authors are employees of Sanofi Pasteur.

Copyright (c) 2020 Yougbare, McTague, He, Choy, Su, Gajewska and Azizi. This is an open-access article distributed under the terms of the Creative Commons Attribution License (CC BY). The use, distribution or reproduction in other forums is permitted, provided the original author(s) and the copyright owner(s) are credited and that the original publication in this journal is cited, in accordance with accepted academic practice. No use, distribution or reproduction is permitted which does not comply with these terms. 